\title{
Benchmarking Knowledge-assisted Kriging for Automated Spatial Interpolation of Wind Measurements
}

\author{
Zlatko Zlatev, Stuart E. Middleton, Galina Veres \\ IT Innovation Centre \\ University of Southampton \\ Southampton, UK \\ $\{z d z, s e m, g v v\} @$ it-innovation.soton.ac.uk
}

\begin{abstract}
We have benchmarked a novel knowledgeassisted kriging algorithm that allows regions of spatial cohesion to be specified and variograms calculated for each region. The variogram calculation itself is automated and spatial regions are created via offline automated segmentation of either expert-drawn Google Earth polygons or NASA altitude data. Our use-case is to create wind interpolation grids for input into a bathing water quality model of microbial contamination. We benchmark our knowledge-assisted kriging algorithm against 7 other algorithms on UK met-office wind data (189 sensors). Our wind estimation results are comparable, but not better than ordinary kriging, but the kriging error maps are much sharper and reflect the known spatial features better. These results are very promising when considering it is an automated approach and allows on-demand datasets to be selected and thus real-time interpolation of previously unknown measurements. Automation is important in progressing towards a pan-European interpolation service capability.
\end{abstract}

Keywords: Data Fusion, Kriging, Spatial Interpolation, Wind Speed, Wind Direction, OGC, WPS

\section{Introduction}

In-situ meteorological sensor measurements are generally recorded by sensor hardware at point locations, requiring some form of spatial interpolation if estimates at other locations are needed. Many spatial interpolation methods exist, both deterministic and geo-statistical, with accuracies dependent on the nature of the observed phenomena, spatial density of sensors, temporal frequency of sampling and the consistency and accuracy of measurement.

In the SANY project [6] we have developed a interpolation algorithm for handling wind measurements. Our use case is to generate spatial grids of estimated wind measurements for continual input into a bathing water quality model [13] and subsequent live prediction of microbial contamination levels of bathing water at specific beaches. Microbial contamination is important information for coast guards when making the decision as to if, and when, to close public beaches. Our algorithms are phenomena independent, and in SANY we have also successfully applied them to air pollution and ground displacement measurements.

We present a knowledge-assisted kriging algorithm applied to historical wind measurements from the UK meteorological office (UKMO) dataset archives [9]. We report cross-validated results for wind speed mean error (ME), root-mean squared error (RMSE) and the range of estimated values. These results are compared directly to results for seven alternative interpolation methods reported in [7] on the same dataset.

Our knowledge-assisted kriging algorithm takes as input known areas of spatial cohesion, either identified by an expert or automatically calculated from the CGIAR-CSI GeoPortal SRTM (90m resolution) Digital Elevation Dataset [8]; multi-region kriging is then used to compute variograms for each region. This algorithm is hosted within an Open Geospatial Consortium (OGC) sensor service framework [5], showing how sensor processing services can be setup to 'play and process' different sensor measurement datasets on-demand.

We outline in section 2 relevant related work, describe our algorithm in section 3 and experimental results in section 4. We discuss and conclude in sections 5 and 6.

\section{Related work}

In addition to ordinary kriging [1], which is outlined in the next section, there are a number of variants such as universal kriging [3], where a trend in data is assumed, and co-kriging [11], where covariates are provided in the same area as the primary sampled measurement to assist in prediction. Our novel multi-region kriging approach is another kriging variant, making use of expert knowledge about the spatial cohesiveness in known regions to improve prediction accuracy.

Many general spatial interpolation techniques [12] exist, with most relevant ones including trend surface analysis, inverse distance weighting, local polynomial interpolation and thin plate spline. Until [7] few works had benchmarked these techniques thoroughly for wind measurement interpolation, and our work builds on these results for direct comparison. 


\section{Knowledge-assisted ordinary kriging}

Kriging [1] is a statistical technique for interpolation or random phenomenon that uses a linear combination of observed values at observed spatial locations to estimate the value at an unobserved location of interest. In contrast to other interpolation techniques like inverse distance weighting and thin plane spline, it uses a model of the phenomenon's spatial correlation encoded as a corrrelogram of a semi-variogram. Also, kriging is an exact interpolator, i.e. it respects the observed values at the observed locations, which differentiates it from interpolation techniques like local polynomial interpolation and trend surface analysis. For the purposes of our work we have used ordinary kriging with a semivariogram phenomenon correlation model. When using a semi-variogram, the spatial correlation between measurement points is quantified by means of a semivariance function (1) where $N(h)$ is the number of pairs of measurement points at distance $\mathrm{h}$ apart and $\mathrm{z}\left(\mathrm{s}_{\mathrm{i}}\right)$ is the observed value.

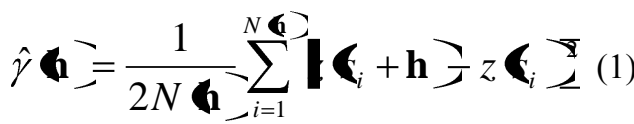

The semi-variance can be a function of both distance and direction, and so it can account for direction-dependent variability (anisotropic spatial pattern). For kriging, a smooth semi-variogram is required and for this purpose a parametric function is used to model the semi-variance for different values of $h$ [2]. These models are fitted to the semi-variance function, or to the experimental semivariogram obtained by averaging the semi-variance function over a set of distance lags, and after a goodness of fit analysis the best model is selected [3] [4]. We refer to this function as a variogram model and the experimental semi-variogram just as experimental variogram. In our kriging implementation with automated variogram model selection we use eight different families of variogram models, namely: spherical, exponential, Gaussian, linear, power, generalised Bessel, sine hole-effect and cosine hole-effect.

It is usual that a phenomenon expert will select a particular variogram model depending on the modeled phenomenon characteristics and fit the selected model to the available observation. This kind of human expert intervention in the interpolation process is expensive and makes the interpolation process phenomena specific. In our solution, ordinary kriging with automated variogram model selection (AVMS), we tackle this problem by automating the variogram model selection.

\subsection{Overall kriging workflow}

The various offline and real-time stages of our knowledgeassisted kriging process is shown in figure 1.

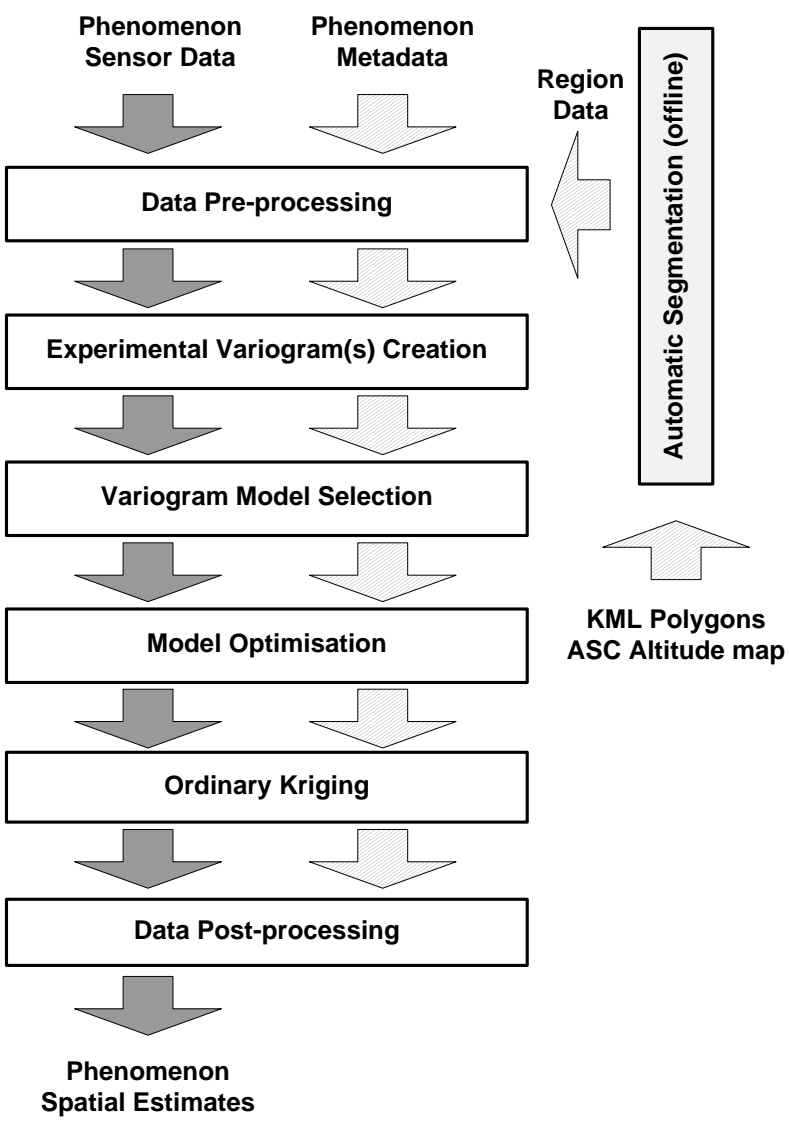

Figure 1. ordinary kriging with automated variogram model selection procedure.

The first stage is the data pre-processing stage, where data cleansing, normalisation and necessary data transformations are performed. The pre-processing stage includes input of knowledge-based descriptions of regions of spatial cohesion, prepared offline using segmentation techniques prior to run-time. In the data post-processing stage, data de-normalisation and reverse transformations are performed. The core ordinary kriging with AVMS stages are the experimental variogram creation, variogram model selection, model optimisation and ordinary kriging. A variogram is created for each region of spatial cohesion. Phenomenon metadata is setup by an expert in a profile configuration.

\subsection{Offline identification of spatially coherent regions}

Region calculation is performed automatically from either expert drawn Google Earth spatial polygons (KML format) or from NASA altitude data (ASC format) from the CGIAR-CSI GeoPortal SRTM (90m resolution) Digital Elevation Dataset [8]. The polygons / altitude maps are rendered as greyscale images and standard image processing techniques (colour reduction, binary mask per colour, pixel blur, labelling and edge identification) used to segment maps into unique regions suitable for input into the kriging process. Region segmentation is executed 
offline, via an automated web service, as part of the initial configuration stage prior to on-demand kriging.

We expect over mountainous grounds the mean daily wind will have lower levels of spatial correlation than over flat land. The land/sea boundary will also have an effect, especially since our sensors are land-based. Figure 2 shows the altitude segmentation, which we found to be somewhat over-segmented and too fine-grained for our wind phenomena. Figure 3 shows the region segmentation for the expert drawn polygons, which produced the best results and are used in the experiments later in this paper.

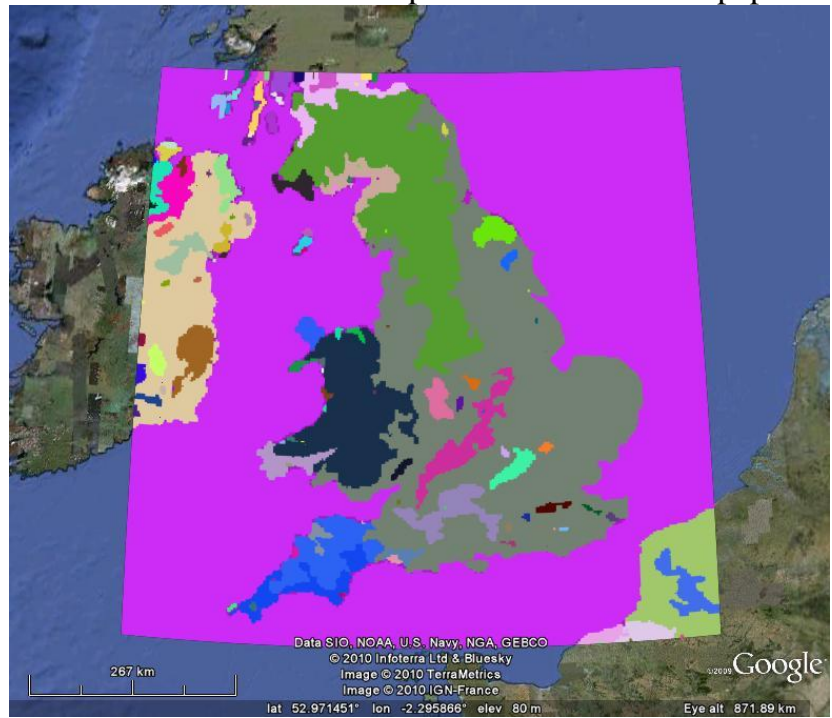

Figure 2. Region segmentation based on CGIAR-CSI GeoPortal SRTM altitude data

The output of the offline region segmentation process are a set of comma separated variable (CSV) maps containing region labels for every interpolation point, and interregion neighbour linkage which is used by the knowledgeassisted ordinary kriging algorithm.

\subsection{Real-time automated variogram model creation}

The most critical part of the experimental variogram creation stage is the selection of lags. Lags need to be selected so they contain an optimal number of points in such a way that physical phenomenon characteristics are not smoothed out but that noise is avoided. Generally the initial slope of the variogram carries the most information so the first few lags shall contain smaller number of points. If no hole-effect is expected the following lags may contain a large number of points, but if hole-effect is expected the lags shall contain a lower number of points so the effect is not smoothed out. The relative number of points in a lag is specified in the metadata supplied to the interpolation procedure by setting the percentile of the semi-variogram function points to be preceding each lag of the experimental variogram. For our experiments with average daily wind speed interpolation we set these percentiles to be as follows: $5,10,15,20,30,40,50,60$, $70,80,90,100$.

In the next stage, the variogram model selection, we fit the eight variogram models pointed out above to the experimental variogram. The model shape is governed by a subset of the following parameters: nugget, correlation range, power, hole and sill. We use a weighted leastsquares fitting method to select a model that best fits the experimental variogram. We introduce phenomenon knowledge by constraining the fitted model types and the parameter values and then, in effect, a variogram model reflecting the characteristics of the phenomenon of interest will be selected.

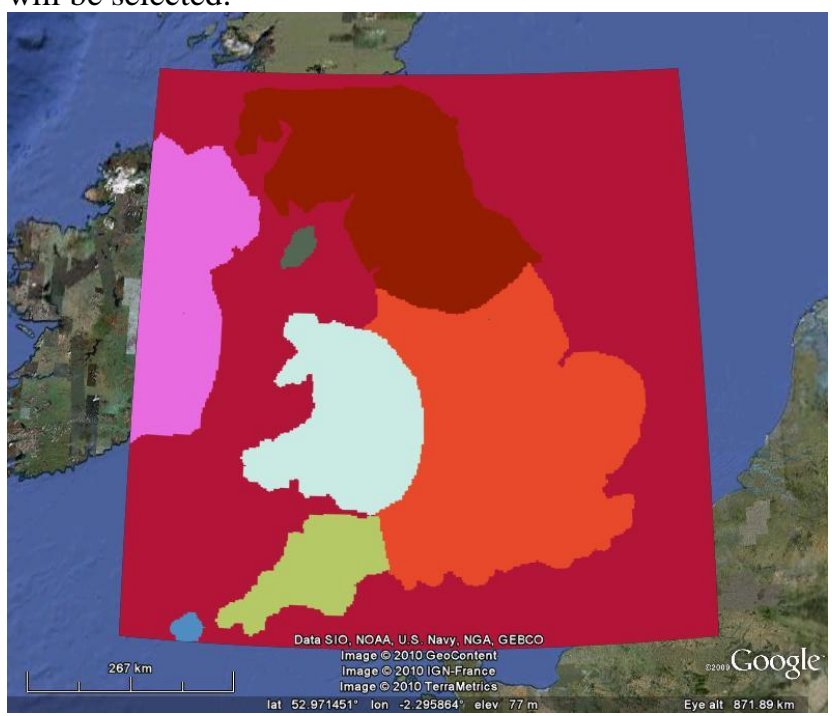

Figure 3. Region segmentation based polygons drawn by an expert for land/water and flat/hilly areas

For daily mean wind speed we expect low levels of noise to be present in the observations because of the averaging used to calculate the means (see section 4.1) and low level of rapid phenomenon fluctuations. So, in the phenomenon metadata we set the nugget to be constrained to maximum of $20 \%$ of the sill. We expect the spatial correlation of the daily mean wind speed to decrease very slowly with increasing the distance, so in the phenomenon metadata we set the lower and upper bounds of the correlation range parameter to be relatively high, respectively $25 \%$ and $75 \%$ of the maximum distance in the experimental variogram. We don't expect a hole effect, so the models with hole effect are not selected.

After selecting the variogram model, parameter optimisation is performed by minimising the the mean error (ME, see equation (2)) and the root mean square error (RMSE, see equation (3)) calculated over 10-folds of cross-validation. We use simplex optimisation with a loss function the sum of ME and RMSE. The phenomenon constraints are reflected in the loss function by adding a high penalty value when the model parameters are outside the specified range.

After the variogram model optimisation stage ordinary kriging with a moving neighbourhood is performed using 
the optimised variogram model and estimate's mean and kriging error is computed. The kriging mean is the estimated value of the phenomenon at the location of interest. The kriging error is a measure of how good the observations' location configuration is for estimation.

Ordinary kriging with a moving neighbourhood is able to pick variations of the phenomenon mean along the interpolated area. As the interpolated area is relatively large in respect to the phenomenon scale we expect variations in the mean and in the phenomenon metadata we have specified; a constrained moving neighbourhood consisting of the 11 closest observations is used. Looking at the observations configuration we inferred that the neighbourhood shall be constrained to about 10 and through experimentation established that the best value is 11. This process can be automated by including an additional parameter to be optimised in the model optimisation stage, which we intend to implement in future versions.

A point map showing region labels for the interpolated area is supplied to our ordinary kriging procedure so we know which region every interpolated point belongs to. The observations belonging to a particular region are used for working out the variogram of that region. If there are not enough observations available for a given region, observations from the neighbouring regions are pulled in order to build the raw variogram. In our ordinary kriging we have the requirement of minimum number observations for variogram estimation to be $\operatorname{argmin}(n *(n-1) / 2>3 *$ $N_{\text {lags }}$ ), where $n$ is the number of observations and $N_{\text {lags }}$ is the number of lags in the variogram, i.e. we have minimum of 3 raw variogram points per lag. Next, when estimating values at unobserved location in e.g. region $\mathrm{A}$, for all the observations values used from region $\mathrm{A}$ we use the variogram of region $\mathrm{A}$, and for all the observations values used from e.g. region $B$ we use a variogram which is the average of variograms A and B. Additionally in the phenomenon metadata we have an inter-region correlation factor, $r$, ranging between 0 and 1 , where 0 indicates minimal correlation between the regions and 1 indicates high correlation. We modulate the averaged inter-region variogram by increasing the variogram values by a quantity equalling $1-r$ times the variogram sill, but up to a value not larger than the sill. If the variogram doesn't have a sill, e.g. the power model, variogram values are increase by a quantity equalling 1-r times the current variogram value. In this way we introduce additional knowledge of factors influencing the phenomenon spatial correlation.

\section{Experiments and results}

In the SANY project we have used our ordinary kriging with AVMS for interpolating micro-scale phenomena, e.g. ground displacement caused by underground tunnelling, and mini to meso-scale phenomena, e.g. wind speed and direction, yielding meaningful results.

In this paper we directly compare the performance of our algorithm against 7 well known algorithms and implementations on a benchmark data set. A definitive work in this context is [7], where the performance of seven interpolation methods are compared on a dataset of daily mean wind speeds obtained from sensor data supplied by the UK meteorological office (UKMO).

Similarly to [7] we have used leave-one-out cross validation and calculated the mean error (ME) and root mean square error (RMSE) as follows:

$$
\begin{aligned}
& \left.M E=\frac{1}{N} \sum_{i=1}^{N}\left[{ }^{\wedge} \boldsymbol{C}_{i}\right) z \boldsymbol{\varepsilon}_{i}\right] \\
& R M S E=\sqrt{\left.\frac{1}{N} \sum_{i=1}^{N}\left[{ }_{z}^{\wedge} \mathbf{C}_{i}\right\rangle z \mathbf{k}_{i}\right]^{2}}
\end{aligned}
$$

where $N$ is the number of validation folds, $\hat{z}_{-}^{-}$is the estimated value and $\mathrm{z}\left(\mathrm{s}_{\mathrm{i}}\right)$ is the observed value.

\subsection{Data}

For out wind speed experiments we used the dataset used in [7] for $27^{\text {th }}$ of March 2001, which was originally obtained from the UKMO. At this date 189 sensors in England, Wales, South of Scotland and Northern Ireland reported at least 12 times, the readings were averaged to obtain daily mean wind speeds. The daily mean wind speeds vary from 2.2 to $13.6 \mathrm{~m} / \mathrm{s}$. Figure 4 depicts the locations and magnitudes of the daily mean wind speed observations, where the size of the dots is relative to the magnitude of the speed.

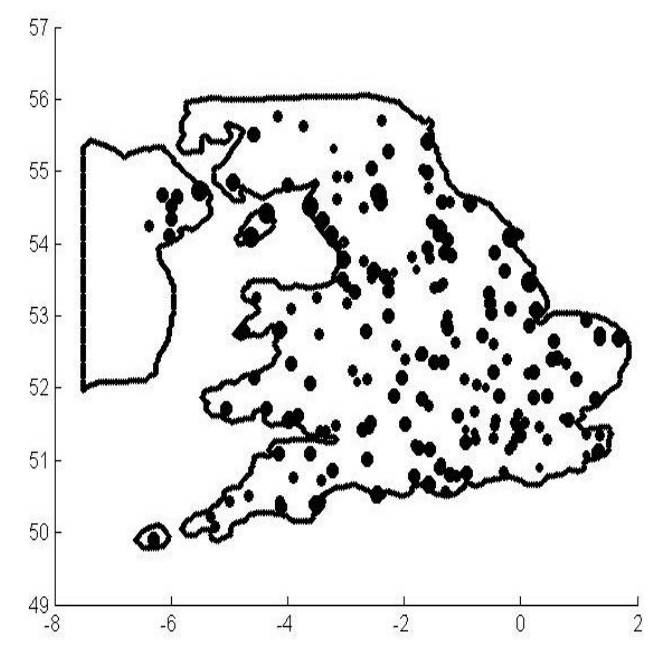

Figure 4. Locations of daily mean wind speed observations for $27^{\text {th }}$ of March 2001.

For our wind direction experiments we used $27^{\text {th }}$ March 2001 data from the UKMO dataset archive [9]. It contains 157 observations with daily mean wind directions. Figure 5 depicts the locations of the daily mean wind directions observations, where the vectors point into the direction of the wind. 
In our experiments we apply our ordinary kriging with AVMS to this data an interpolate on a grid covering England, Wales, South of Scotland and Northern Ireland, having resolution of $5 \mathrm{~km}$ by $5 \mathrm{~km}$. For daily mean wind speed we compare our results against the results published in [7]. In [7] there are no results present for daily mean wind directions so we just state the results from our experiments and show the relevant visualizations.

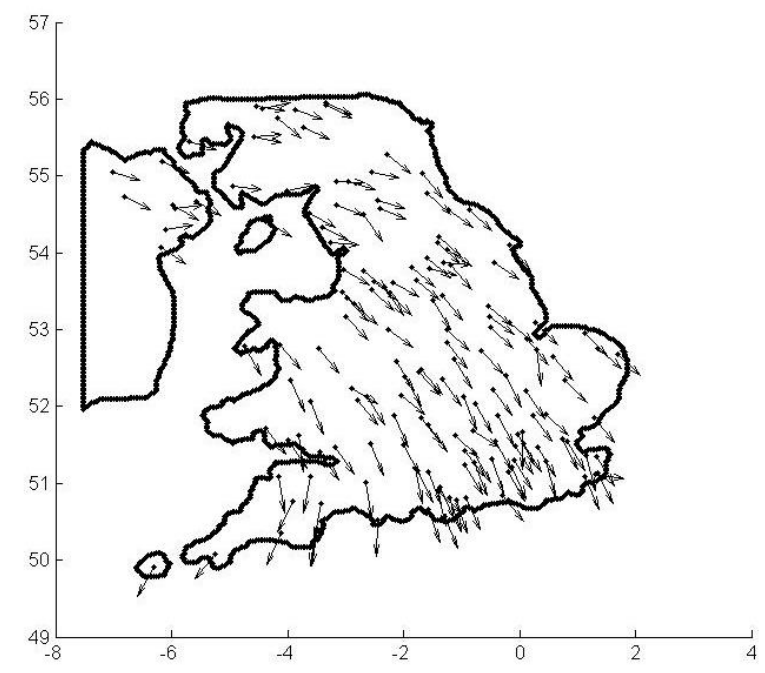

Figure 5. Locations of daily mean wind direction observations for $27^{\text {th }}$ of March 2001 .

\subsection{Daily mean wind speed estimates benchmarking}

In this experiment we compare our base ordinary kriging with AVMS algorithm against the 7 algorithms evaluated in [7]. We set the algorithm with the following parameters:

1. Experimental variogram lags percentiles: 5, 10, 15, 20, 30, 40, 50, 60, 70, 80, 90, 100.

2. Nugget boundaries: 0 to $20 \%$ of the sill.

3. Correlation range boundaries: $25 \%$ to $75 \%$ of the maximum variogram distance.

4. Variogram models to use: spherical, exponential, Gaussian, linear, power or generalised Bessel.

\section{Ordinary kriging (no regions)}

The surface produced is consistent with the input values over land, see figure 6 . Looking at the surface over land, where the sensors are located, high wind speeds are accurately estimated over the north, the north-east and north-west of England, and also over the Isle of Man. Places of low wind speed are accurately estimated over the Midlands, and the west and south-east of England. The estimated surface is very similar to the surfaces shown in [7]. Similarly, the kriging error map over land matches closely the corresponding maps in [7]. The kriging error is small where we have high concentration of observations and larger where there are smaller number of observations. The RMSE, ME and the min and max interpolated values suggest that the performance of ordinary kriging with
AVMS is slightly worse than the ordinary kriging in [7] and slightly better than universal kriging and local polynomial (see Table 1). It should be noted that ordinary kriging with AVMS needs only a minimal setup from the user, unlike [7].

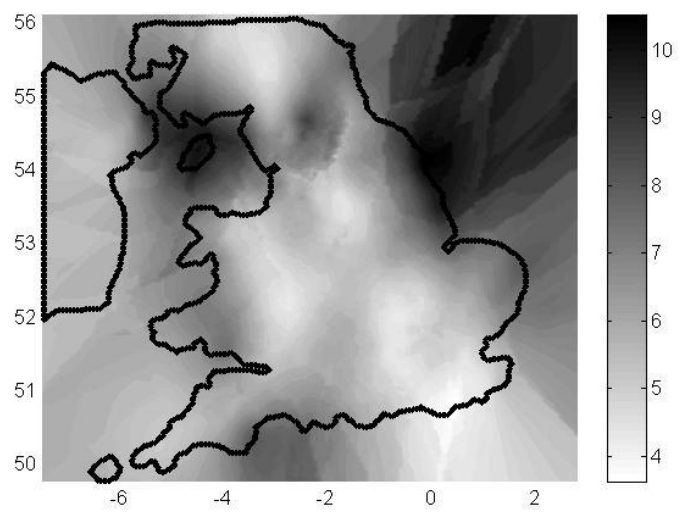

Figure 6. Estimated daily mean winds speed - ordinary kriging with AVMS and no region

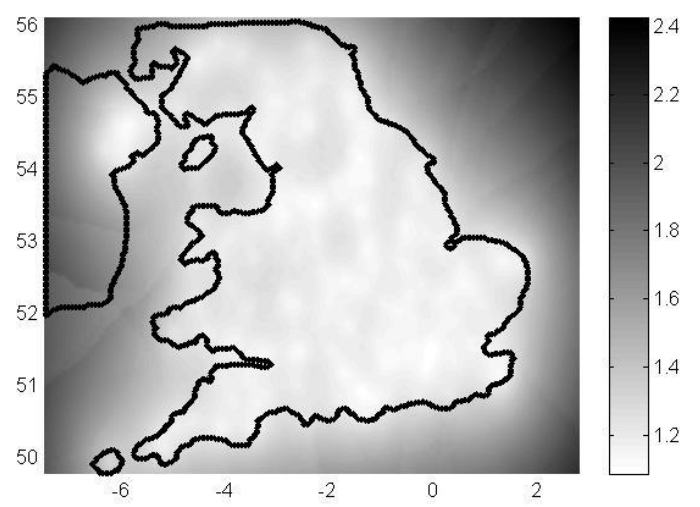

Figure 7. Kriging error of estimated wind speed - ordinary kriging with AVMS and no region

\section{Ordinary kriging (coastline regions)}

Over the sea the estimates are in all probability not reliable as this area is not covered by any of the observations. Supplying expert-drawn regions can help in this case. Expecting different behaviour of the interpolated phenomenon over land and over sea we can perform landsea segmentation and supply region information to our ordinary kriging algorithm.

For our next experiment we have performed coastline segmentation using Google Earth drawn polygons and supplied the resulting regions to our ordinary kriging with AVMS. The inter-region correlation factor $r$ was set to 0.1 to indicate that there are minimal similarities in the way the different regions, sea and land, influence the phenomenon behaviour.

The wind speed estimates change only slightly as all of the observations are over land. The only notable difference here are the estimates over the Isle of Man, see figures 6 and 8 , where more accurate high values are predicted as 
the mainland observations interference is diminished by the low inter-region correlation factor. This is also reflected in the algorithm performance metrics in Table 1. The kriging error for the Isle of Man is very different from the kriging error without regions. Compare figures 9 and 7. There is a clear jump in the kriging error on the landsea border indicating that, as we don't have observations in the sea, the wind speed estimates over sea are unreliable.

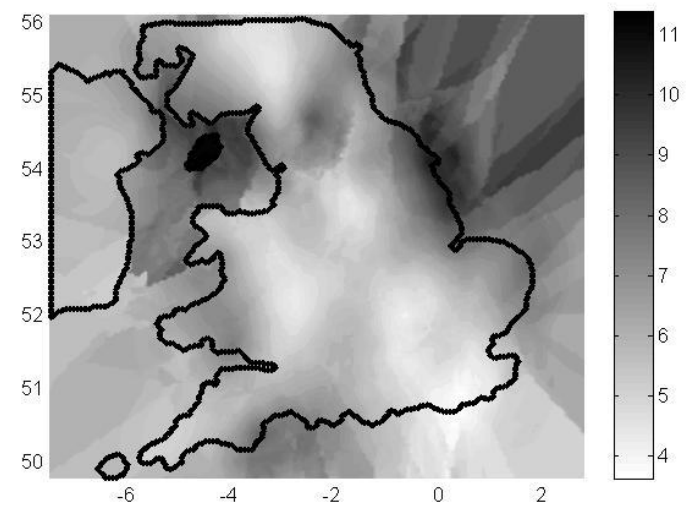

Figure 8. Estimated daily mean winds speed - ordinary kriging with AVMS and coastline regions

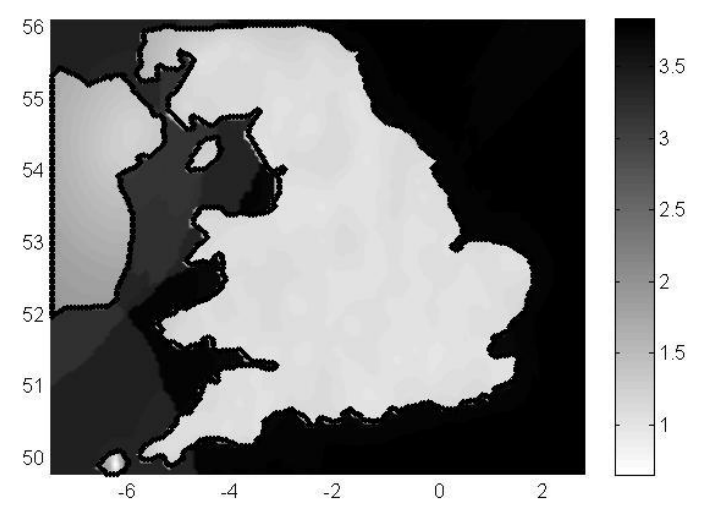

Figure 9. Kriging error of estimated winds speed ordinary kriging with AVMS and coastline regions

\section{Ordinary kriging (regions for flat/hilly areas)}

For our last experiment regarding wind speed we performed further segmentation to separate land areas with different terrain topology, i.e. flat land, hilly land, mountainous land. We used a higher inter-region correlation factor of 0.9 since regions are in-land.

With this segmentation the performance of ordinary kriging with AVMS is improved and is comparable to ordinary kriging in [7] (see Table 1). The bias, ME, is decreased as is the RMSE (see figure 10). The minimum and maximum of the estimated values are closer to those present in the raw data.

The kriging error map, figure 11, shows distinct pattern in the different regions. For example the kriging error over the Midlands, South and South-East region is very smooth, which we interpret as not needing very dense observations to achieve good estimates in this region. In contrast the spotty kriging error pattern in the northern region, and in Wales and south-west of England, we interpreted as needing much more dense observations to achieve good estimates in these regions.

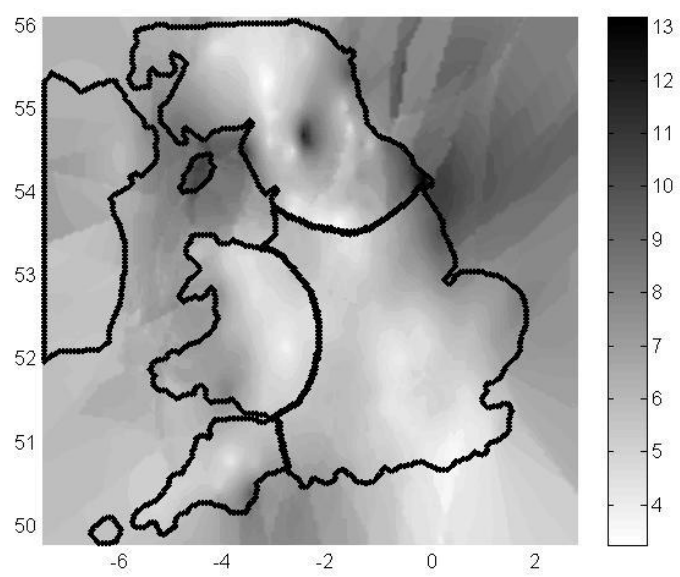

Figure 10. Estimated daily mean winds speed - ordinary kriging with AVMS with flat/hilly regions

\begin{tabular}{|l|l|l|l|}
\hline Method & $\begin{array}{l}\text { Number of } \\
\text { Regions }\end{array}$ & $\begin{array}{l}\text { Inter-region } \\
\text { correlation }\end{array}$ & $\begin{array}{l}\text { Exec-time } \\
\text { (mins) }\end{array}$ \\
\hline Kriging_1 & 0 & n/a & 1 \\
\hline Kriging_2 & 4 coastline & 0.1 & 8 \\
\hline Kriging_3 & 7 flat/hilly & 0.9 & 10 \\
\hline All methods use ordinary kriging with AVMS \\
Computer spec : 2 CPU's (2.4 GHz) with 4 Gbyte RAM \\
\hline
\end{tabular}

\begin{tabular}{|l|l|l|l|l|}
\hline \multicolumn{1}{|c|}{ Method } & $\begin{array}{l}\text { Min } \\
\text { estimate } \\
\mathbf{m} / \mathbf{s}\end{array}$ & $\begin{array}{l}\text { Max } \\
\text { estimate } \\
\mathbf{m} / \mathbf{s}\end{array}$ & $\begin{array}{l}\text { RMS } \\
\mathbf{E} \\
\mathbf{m} / \mathbf{s}\end{array}$ & $\mathbf{M E}$ \\
\hline Kriging_1 & 3.6 & 10.7 & 1.67 & -0.03 \\
\hline Kriging_2 & 3.6 & 11.6 & 1.67 & -0.03 \\
\hline Kriging_3 & 3.24 & 13.40 & 1.60 & -0.01 \\
\hline Cokriging [7] & 2.6 & 13.6 & 1.47 & -0.01 \\
\hline $\begin{array}{l}\text { Ordinary } \\
\text { kriging [7] }\end{array}$ & 2.6 & 13.4 & 1.61 & -0.01 \\
\hline $\begin{array}{l}\text { Local } \\
\text { polynomial [7] }\end{array}$ & 0.1 & 11.9 & 1.69 & 0.01 \\
\hline $\begin{array}{l}\text { Universal } \\
\text { kriging [7] }\end{array}$ & 3.2 & 9.8 & 1.71 & 0.01 \\
\hline IDW [7] & 3.1 & 12.1 & 1.74 & -0.09 \\
\hline TPS [7] & 1.5 & 13.8 & 1.89 & -0.05 \\
\hline TSA [7] & 4.6 & 9.2 & 1.93 & -0.02 \\
\hline
\end{tabular}

Table 1. Comparison of knowledge-assisted kriging to benchmark interpolation results reported in [7]

\subsection{Daily mean wind direction estimation}

Using the same ordinary kriging with AVMS setup as for the wind speed experiments we ran ordinary kriging with AVMS (no regions) on the UKMO dataset for $27^{\text {th }}$ March 
2001. In our implementation of ordinary kriging we have a special procedure [10] for handling periodic values like wind direction, which includes vector rotation and Cartesian transformation and simulation.

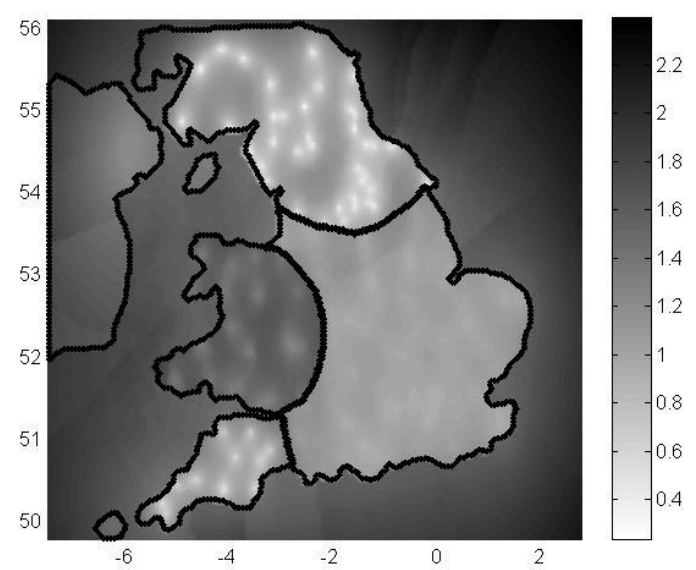

Figure 11. Kriging error of estimated winds speed ordinary kriging with AVMS with flat/hilly regions

Estimated wind direction vectors are visualised in figure 12. This is done on a 25 by 25 points grid to make visualisation easier. The ME and RMSE are given in Table 2. We find the ME of -0.60 degrees and RMSE of 13.66 degrees in relative terms are very low considering worse case could be 180 degrees. The mean relative absolute error (MRAE) is 6\%. For our wind speed experiments MRAE varies from $22 \%$ to $23 \%$. For wind speed we calculate MRAE as:

$\left.\left.M R A E=\frac{1}{N} \sum_{i=1}^{N}\left[\mid z \boldsymbol{C}_{i}\right) z \boldsymbol{\zeta}_{i}\right] / z \boldsymbol{\zeta}_{i}\right]$

and for wind direction we calculate MRAE as:

$\left.M R A E^{(\text {periodic })}=\frac{1}{N} \sum_{i=1}^{N}\left[\operatorname{norm}\left(\mid z \hat{q}_{i}-z\right]\right) / 180\right]$

where $\mathrm{N}$ is the number of validation folds, $z \mathbf{C}_{-}$is the estimated value, $\mathrm{z}\left(\mathrm{s}_{\mathrm{i}}\right)$ is the observed value, $|$.$| is the$ absolute value and norm(.) yields a normalised wind direction in the range of -180 to +180 .

The wind direction kriging error is shown in figure 13, with higher absolute values since we do not normalize keeping a range of 0 to 360 . Kriging error quantifies the suitability of the observations configuration for estimating at a particular location and is not a measure of the estimation error in itself.

\begin{tabular}{|c|l|l|}
\hline Method & $\begin{array}{l}\text { ME } \\
\text { degrees }\end{array}$ & $\begin{array}{l}\text { RMSE } \\
\text { degrees }\end{array}$ \\
\hline Ordinary kriging with AVMS & -0.60 & 13.66 \\
\hline
\end{tabular}

Table 2. Estimated daily mean wind direction ME and RMSE values - ordinary kriging with AVMS.

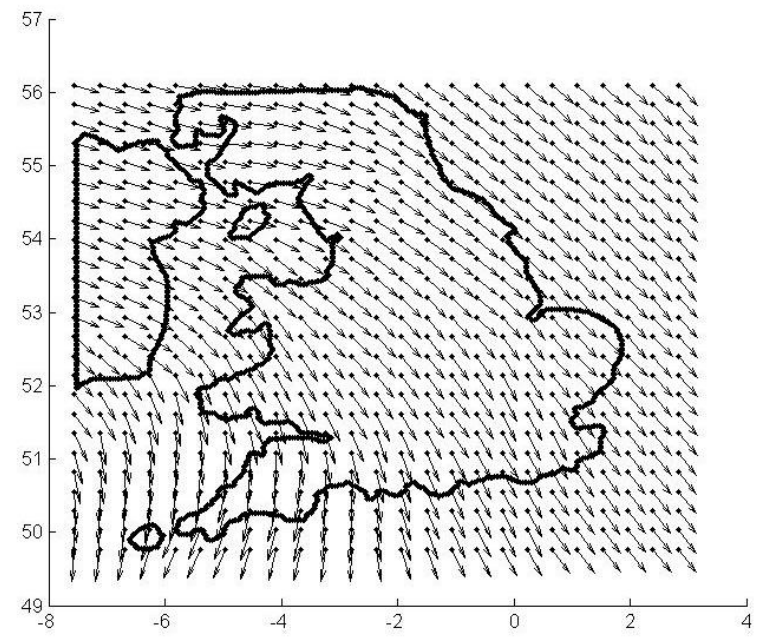

Figure 12. Estimated daily mean winds directions ordinary kriging with AVMS no region

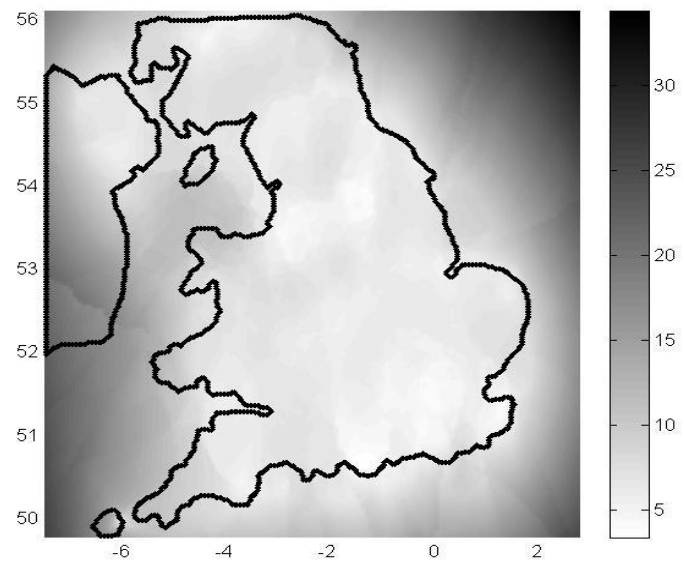

Figure 13. Kriging error for estimated daily mean winds directions - ordinary kriging with AVMS no regions

We are unaware of any other benchmark wind direction interpolation results so publish here in the hope that future work can compare against our results.

\section{Discussion}

Our experiments have investigated methods to automatically select variogram models and the impact using different levels of expert knowledge, about the phenomenology and the spatial area of interest, can have on estimation error. This automated approach is in contrast to existing methods [7] that rely on manual variogram tuning by experts. We think our approach offers a realistic route to providing on-demand kriging services that can interpolate measurement datasets selected by users that are unknown until run-time. This level of automation is becoming more important as we see European environment agencies sharing increasing amounts of sensor data under the INSPIRE directive [15]. Such automation, and a move away from manual tuning and configuration, increases scalability and could allow 
truly dynamic pan-European interpolation for wind measurements and other phenomenology.

The performance of our ordinary kriging with AVMS algorithm is slightly worse than that reported by ordinary kriging in [7] with expert tuned variogram. Partly this is due to the experts skill in manually tuning the variogram. However our implementation is also only isotropic, which accounts for some of the performance compared against the anisotropic ordinary kriging algorithm in [7]. We intend to implement anisotropic kriging in future releases. Where our knowledge-assisted approach offers the most improvement, compared to basic ordinary kriging, is in the enhanced spatial definition, and therefore confidence, in the kriging error maps. This is most clear in areas where no sensors are located (i.e. offshore in the sea). This result can be applied more widely to other spatial features than hills and coastlines, at a variety of different spatial scales, such as building footprints and river outlets. Though not the focus of this wind interpolation paper we, have successfully interpolated measured phenomenon including ground displacement, water salinity and turbidity.

\section{Conclusion}

We have benchmarked a novel knowledge-assisted kriging algorithm that allows regions of spatial interest to be specified and variograms calculated for each region. The variogram calculation itself is automated and phenomenon specific metadata allows us to configure kriging for more than just wind phenomenon. Spatial regions are created automatically offline by segmentation of either expertdrawn Google Earth polygons or NASA altitude data.

Our use-case is to create wind interpolation grids for input into a bathing water quality model of microbial contamination, for subsequent decision support for beach attendants for bathing risk assessment.

We benchmark our knowledge-assisted kriging algorithm against 7 other algorithms using the same UK met-office wind measurement dataset reported in [7]. Wind speed estimation results are comparable, but not better than ordinary kriging, but the kriging error maps are much sharper and reflect the known spatial features better. We provide results for wind direction interpolation also.

These results are very promising when considering it is an automated approach and allows on-demand datasets to be selected and real-time interpolation of previously unknown measurements. Automation is important to move towards a pan-European interpolation service capability making use of European environment agency data shared in compliance with the European INSPIRE directive [15].

This work was funded by the European Commission's IST Programme under contract FP6-IST 0033564 SANY [6]

\section{References}

[1] D.G. Krige, Two-dimensional weighted average trend surfaces for ore-evaluation, Journal of the South
African Institute of Mining and Metallurgy 66: 13-38, 1966

[2] N.A.C. Cressie, Fitting variogram models by weighted least squares, Journal of the International Association for Mathematical Geology 17: 653-702, 1985

[3] N.A.C. Cressie, Statistics for Spatial Data, John Wiley and Sons: New York, 1993

[4] P.A. Burrough and R.A. McDonnell, Principles of Geographical Information Systems, Clarendon Press: Oxford, 1998

[5] S.E. Middleton (ed) et al, SANY Fusion and Modelling Architecture, OGC Discussion paper 10-001, 2010

[6] SANY project, http://www.sany-ip.eu

[7] W. Luo, M.C. Taylor and S.R. Parker, A comparison of spatial interpolation methods to estimate continuous wind speed surfaces using irregularly distributed data from England and Wales, Int. J. Climatol, 28: 947-959, 2008

[8] CGIAR-CSI GeoPortal SRTM (90m resolution) Digital Elevation Dataset, http://srtm.csi.cgiar.org

[9] UK Met Office - MIDAS Land Surface Observation Stations Data, http://badc.nerc.ac.uk/data/ukmo-midas/

[10] Z. Zlatev, S.E. Middleton and G. Veres, Ordinary kriging for on-demand average wind interpolation of insitu wind sensor data, EWEC 2009, France, March 2009

[11] A.G. Journel, C. Huijbregts, Mining Geostatistics, Academic Press: New York, 1978

[12] N.S. Lam, Spatial interpolation methods: a review, The American Cartographer 10: 129-135, 1983

[13] Z.A. Sabeur (ed) et al, Development of environmental information tools for the prediction of water quality risks in bathing waters, Final Report to the Environment Agency of England and Wales, Interreg ICREW project, Pilot Action 4: Forecasting bathing water quality. Final Technical Report/FINAL. 21st June 2006, pp128, 2006

[14] R.B. Stull, Meteorology for Scientists and Engineers, 2nd Edition, Earth Sciences series, Cengage Learning Publisher. ISBN-13: 9780534372149, 1999

[15] INSPIRE directive, Directive 2007/2/EC of the European Parliament, 14 March 2007 